\title{
Glucocorticoid response mapped
}

Glucocorticoids are a mainstay treatment for a number of rheumatic diseases, and yet the mechanisms underlying their immunoregulatory effects remain partially understood. To address this knowledge gap, researchers have developed a pathwaylevel map of the genomic response of different cell types to glucocorticoids.

"Within each molecular pathway in this map, we can zoom in to look at the specific genes that are affected," explains Luis Franco, corresponding author of the study. "Our hope is that this map will enable other investigators to explore the response to glucocorticoids in their cell of interest."

The researchers began by using RNA sequencing to simultaneously measure the response to glucocorticoids of every gene in the human genome. "We chose to go beyond mixed cells, to study nine specific cell types: four immune (B cells, CD4 ${ }^{+} \mathrm{T}$ cells, neutrophils, and monocytes) and five nonimmune (osteoblasts, myoblasts, fibroblasts, preadipocytes, and endothelial cells)," explains Franco.

They found that the glucocorticoidmediated transcriptional response was cell type-dependent. Of the 9,457 genes that were differentially expressed in at least one cell type, nearly half were responsive to treatment in only one cell type, and only 25 genes were responsive in all cell types. Furthermore, the responses of particular genes were cell typedependent. "These findings suggest that classifying genes as glucocorticoidinduced or glucocorticoid-repressed, as is common in the literature, should only be done in the context of a specific cell type."

Focusing on B cells, they found that glucocorticoids affect two important pathways: B cell receptor and Toll-like receptor 7 signalling. "Our findings suggest that functional impairment of signalling through these two pathways could be a major reason why glucocorticoids are effective in the treatment of autoimmune diseases".

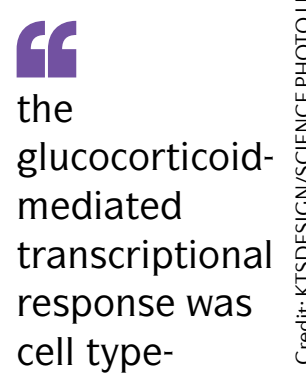

\section{dependent}

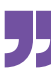

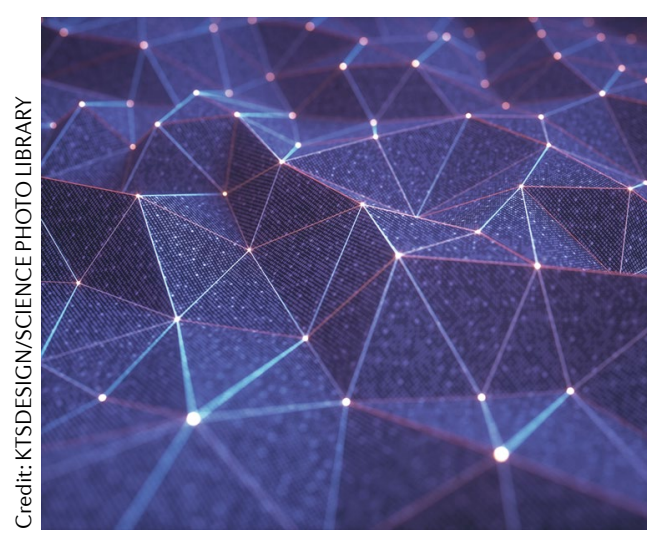

"Now that we have a better idea of the 'normal' human response to glucocorticoids, our immediate plan is to apply a similar approach to understanding the response in patients with autoimmune diseases," says Franco. "A better understanding of what glucocorticoids really do should eventually pave the way to equally efficacious but safer therapeutic alternatives."

\section{Jessica McHugh}

ORIGINAL ARTICLE Franco, L. M. et al. Immune regulation by glucocorticoids can be linked to cell type-dependent transcriptional responses. J. Exp. Med. 216, 384-406 (2019)

\section{Cholesterol test for T cells}

New research published in Nature Communications identifies a regulatory mechanism to account for the switching of IFN $\gamma$ production to IL-10 production by $T$ cells, a step that is thought to be important to limit inflammation and the progression of inflammatory diseases such as rheumatoid arthritis (RA).

"We tried to better understand which signals regulate this switch," explains Esperanza Perucha, co-corresponding author of the new study. "We discovered that cholesterol homeostasis regulates IL-10 expression in human $\mathrm{CD}^{+}{ }^{+} \mathrm{T}$ cells."

To mimic switching in vitro, the researchers stimulated $T$ cells with anti-CD3 and anti-CD46 antibodies, a culture condition that initially results in IFN $\gamma$ and IL-2 production. Accumulation of IL-2 in such cultures then prompts IFN $\gamma^{+}$T cells to produce IL-10, and then in a final phase these cells are fully switched and produce IL-10, but not IFN $\gamma$.

25-HC was
shown to
reduce
expression of
-Maf

By comparing gene expression of cells from each of these three stages of the culture and then running pathway analyses, the researchers found a strong association between the cholesterol biosynthesis pathway and the licensing of IFN $\gamma^{+}$ T cells to switch to IL-10 production.

To functionally validate this finding, the researchers inhibited cholesterol biosynthesis in vitro with the synthetic statin atorvastatin, which prevented IFN $\gamma^{+}$T cells from producing IL-10. A similar block to switching was noted when the cultures were treated with the cholesterol derivative 25-hydroxycholesterol (25-HC), and 25-HC was shown to reduce expression of c-Maf, the master transcription factor of IL-10 production.

"These findings provide a molecular framework for the so-called 'lipid paradox', in which high levels of systemic cholesterol have been linked

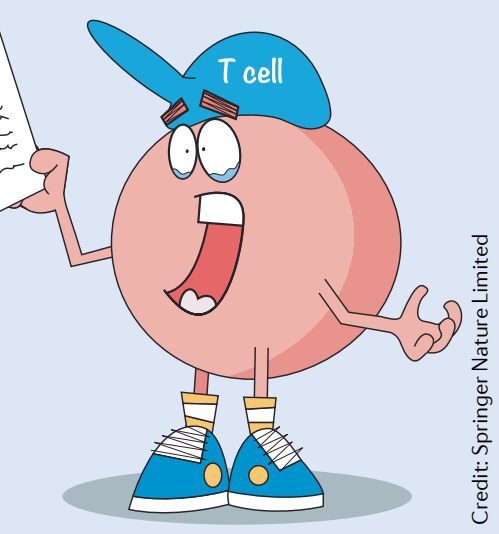

to remission in RA," says Andrew Cope, co-corresponding author of the study.

As preliminary evidence, the researchers also show a positive association between expression of $\mathrm{CH} 25 \mathrm{H}$ (which encodes the enzyme that converts cholesterol to 25-HC) and progression to RA in a small cohort of patients with autoantibody-positive arthralgia.

Nicholas J. Bernard

ORIGINAL ARTICLE Perucha, E. et al. The cholesterol biosynthesis pathway regulates IL-10 expression in human Th1 cells. Nat. Commun. 10 , 498 (2019) 